\title{
A New HI Ring: LGG 138
}

\author{
David G. Barnes \\ Australia Telescope National Facility, CSIRO, PO Box 76, Epping, NSW 2122, Australia \\ dbarnes@atnf.csiro.au
}

\begin{abstract}
A complete ring of neutral hydrogen gas (HI) in the LGG 138 group of galaxies has been found. The Hi mass of the ring is greater than $10^{9} h_{75}^{-2} M_{\odot}$, and the gas appears to be rotating with a projected circular speed of $\sim 200 \mathrm{~km} \mathrm{~s}^{-1}$. Two bright galaxies are enclosed by the ring, both having radial velocities consistent with membership of the group. Faint stellar emission extends to the radial distance of the Hi ring, where a small but distinct colour discontinuity of between $0 \cdot 05$ and $0 \cdot 20$ magnitudes is detected. Three simple models for the formation of the system are briefly described, the most likely appearing to be a past gas-sweeping collision between one of the two bright galaxies and an outside intruder, with the colour break being partly due to an expanding density wave that is triggering star formation, and partly to a different stellar population that has been collected from the outskirts of the intruder.
\end{abstract}

Keywords: galaxies: kinematics and dynamics — galaxies: interactions — galaxies: clusters: individual (LGG 138)

\section{Introduction}

The distribution and kinematics of neutral gas in galaxy groups can be studied using $21 \mathrm{~cm}$ emission-line observations of neutral hydrogen (HI). In particular, since $\mathrm{HI}$ is generally the most extended disk tracer in normal galaxies, high-resolution $\mathrm{HI}$ observations can be used to search for evidence of strong, mild and even weak galaxy interactions within groups. Some systems in which HI studies have revealed important interaction physics include Stephan's Quintet, for which Shostak et al. (1984) showed that the bulk of the Hi lies outside the optical galaxies at all velocities, Hickson compact group (HCG) 18, in which Williams \& van Gorkom (1988) found $\mathrm{HI}$ smoothly distributed over a region twice the optical size of the group, and the Grus Quartet, in which Koribalski (1996) found a number of tidal tails tracing possible baryonic streams between the constituent galaxies.

Most previous Hi studies of small groups of galaxies have concentrated on compact groups of galaxies that satisfy the Rose (1977) or Hickson (1982) criteria. To commence an investigation into a possible relationship between these compact groups and somewhat looser groups - examples being those selected by Garcia (1993), Nolthenius (1993) and Fouqué et al. (1992) - a preliminary neutral gas imaging study of five heterogeneous groups was undertaken by Barnes (1998). One of the groups selected for imaging, LGG 138 (Garcia 1995), turned out to be an intriguing system: two galaxies already identified as group members were found to be encircled by a hollow ring of Hi. The ring differs from many previously known Hi rings in that stellar material is clearly associated with parts of the ring.

\section{Observations and Basic Properties of Ring}

Observations of the $21 \mathrm{~cm}$ HI line in the LGG 138 group were acquired with the Australia Telescope (AT) Compact Array initially in a moderately compact configuration with a maximum baseline of $750 \mathrm{~m}$ in November 1996, and later in an additional $750 \mathrm{~m}$ configuration (March 1997) and three 1500 m configurations (August and September 1997) which sampled higher spatial frequencies on the sky. The AT correlator was programmed to record XX and YY correlations between all telescope pairings, over a bandwidth of $16 \mathrm{MHz}$. In this mode, each correlator spectrum has 512 equally-spaced channels. Consequently, the channel width was $31 \cdot 25 \mathrm{kHz}$, corresponding to a velocity channel width of $6 \cdot 6$ $\mathrm{km} \mathrm{s} \mathrm{s}^{-1}$ in the rest frame of the observations. The total effective on-source integration time was $48 \mathrm{hr}$, and the combined data from the multiple array configurations gave excellent coverage of the $u v$ plane. The data were calibrated, continuumsubtracted and imaged in MIRIAD, and for naturally weighted visibilities yielded channel maps with root mean square noise fluctuations of $0.75 \mathrm{mJy}^{\text {beam }}{ }^{-1}$, and a synthesised beam of dimensions $70^{\prime \prime} \times 35^{\prime \prime}$.

In addition to the Hi data, optical images of the ring were acquired using the Mount Stromlo Observatory 40 inch telescope at Siding Spring Mountain in July 1997. Continuum images in the $B, V$ and $R$ bands were made using a $2048 \times 2048$ element, thinned, charge-coupled device camera with a pixel scale of $0 \cdot 6^{\prime \prime}$ pixel $^{-1}$ when mounted on the telescope. Dark frame subtraction, flat-fielding and calibration of the data was applied using standard procedures in IRAF. 


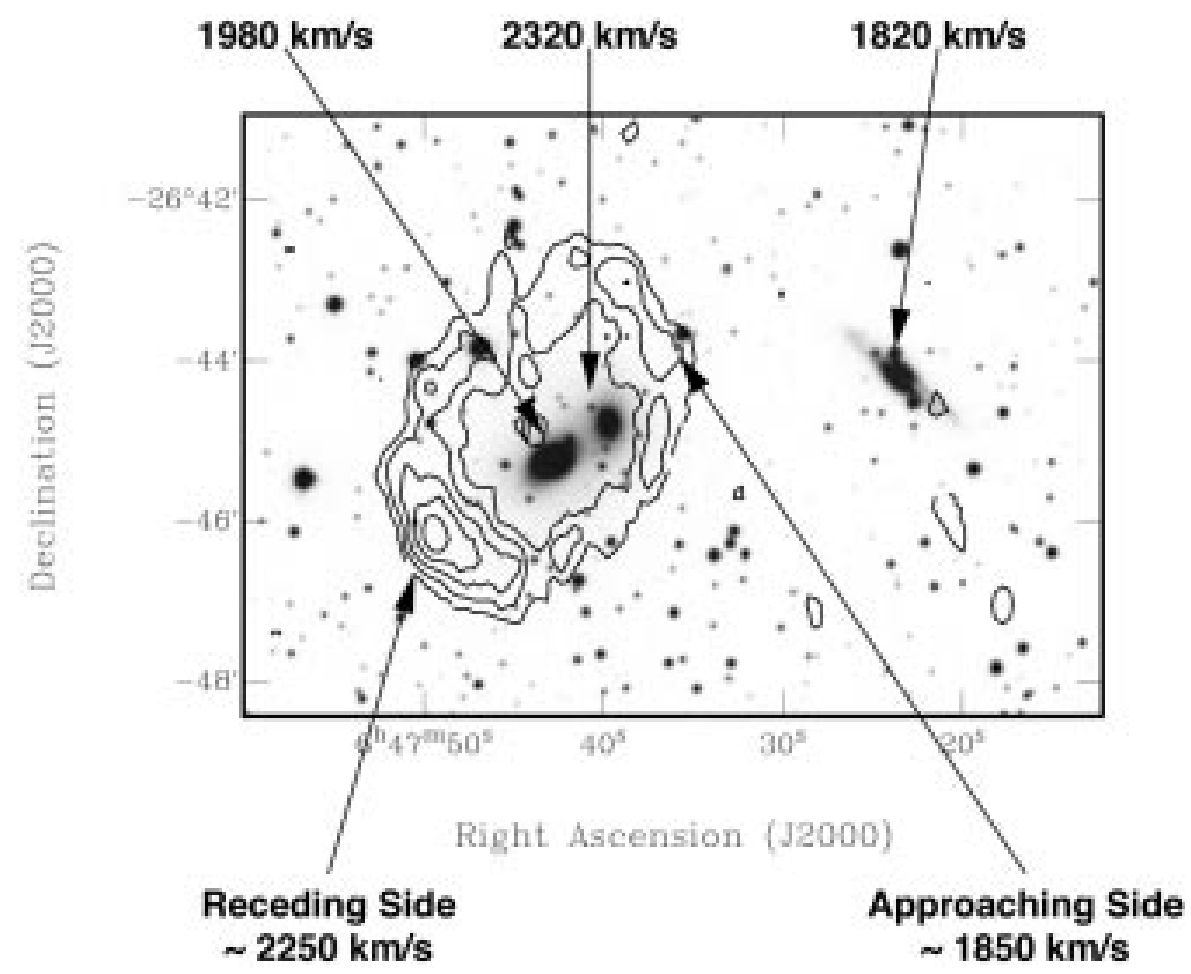

Figure 1-Total Hi column density contours of the LGG 138 ring, at levels of $1,2,3,4$ and $5 \times 10^{20} \mathrm{~cm}^{-2}$, overlaid on a $V$ band continuum image showing the three galaxies NGC $2293 / 2292 / 2295$, listed in order from east to west. The optical velocities of the galaxies are indicated above the figure; intensity-weighted mean Hi velocities are given below the figure for the kinematically-opposite edges of the ring.

The NGC 2292/2293 pair of galaxies was the principal target for the extended integration on LGG 138 obtained with the AT Compact Array. Figure 1 shows the distribution of neutral hydrogen gas relative to the $V$ band optical emission of these two galaxies. Figure 2 shows the same distribution overlaid on a difference image representing $B-R$ colour. It is clear that the $\mathrm{HI}$ is arranged in a large scale ring surrounding the pair, and furthermore, aligns exactly with a distinct colour break in the stellar populations in the south western arc of the ring.

The observations show the HI ring in LGG 138 to be in large scale rotation with a maximum projected speed of $200 \mathrm{~km} \mathrm{~s}^{-1}$, or in expansion with a projected expansion speed of $200 \mathrm{~km} \mathrm{~s}^{-1}$, or, as is more likely, a combination of both rotation and expansion. At an assumed distance of $27 h_{75}^{-1} \mathrm{Mpc}$, the major and minor projected diameters of the ring are $\sim 30 h_{75}^{-1}$ and $\sim 21 h_{75}^{-1} \mathrm{kpc}$ respectively. The Hi mass of the ring, under the usual assumptions, is $1.9 \times 10^{9} h_{75}^{-2}$ $M_{\odot}$ - typical of the quantity of neutral hydrogen bound to an early-type spiral galaxy (Roberts \& Haynes 1994). The ATCA observations reach a $3 \sigma$ column density sensitivity of $1.4 \times 10^{19} \mathrm{~cm}^{-2}$ per $13.2 \mathrm{~km} \mathrm{~s} \mathrm{~s}^{-1}$ channel, and with no detection of $\mathrm{HI}$ at this level directly associated with the optical galaxies, the Hi masses of the encircled galaxies
2292 NGC and 2293 are constrained to be less than $10^{7} h_{75}^{-2} M_{\odot}$ each for $10 \sigma$ detections extending over $150 \mathrm{~km} \mathrm{~s}^{-1}$. The spatial Hi distribution is consistent with the ring actually being narrower than the synthesised beam, i.e. the ring thickness $\delta r \lesssim 4 \mathrm{kpc}$, or alternatively, $\delta r / r \lesssim 0 \cdot 25$, where $r$ is the radius of the ring. For column densities above $10^{20} \mathrm{~cm}^{-2}, \delta r / r \simeq 0 \cdot 3$, and thus the ring is believed to be radially thin.

Without knowing the true physical shape of the ring, it is difficult to determine the kinematics and hence dynamics of the NGC 2292/2293 system. However, one particularly simple model can be tested: if the ring is intrinsically circular, or nearly circular, then the projected eccentricity $(e=0 \cdot 71)$ can be used to estimate the inclination angle, which is found to be of order $45^{\circ}$. The radius of this circular ring is $\sim 15 \mathrm{kpc}$, and the ring rotates with a maximum (de-projected) circular speed of $280 \mathrm{~km} \mathrm{~s}^{-1}$. In these conditions, the rotation period of the ring is $330 \mathrm{Myr}$ - only 50 per cent higher than that of the Sun around the centre of the Galaxy. The mass within the ring needed to support the rotation is $2.7 \times 10^{11} h_{75}^{-1} M_{\odot}$. The total (Cousins) blue luminosities of 2292 and 2293 are 11.8 and 11.7 (Lauberts \& Valentijn 1989), yielding a total integrated blue luminosity from the system of $4.6 \times 10^{10} h_{75}^{-2} L_{\odot}$, and a total mass to 


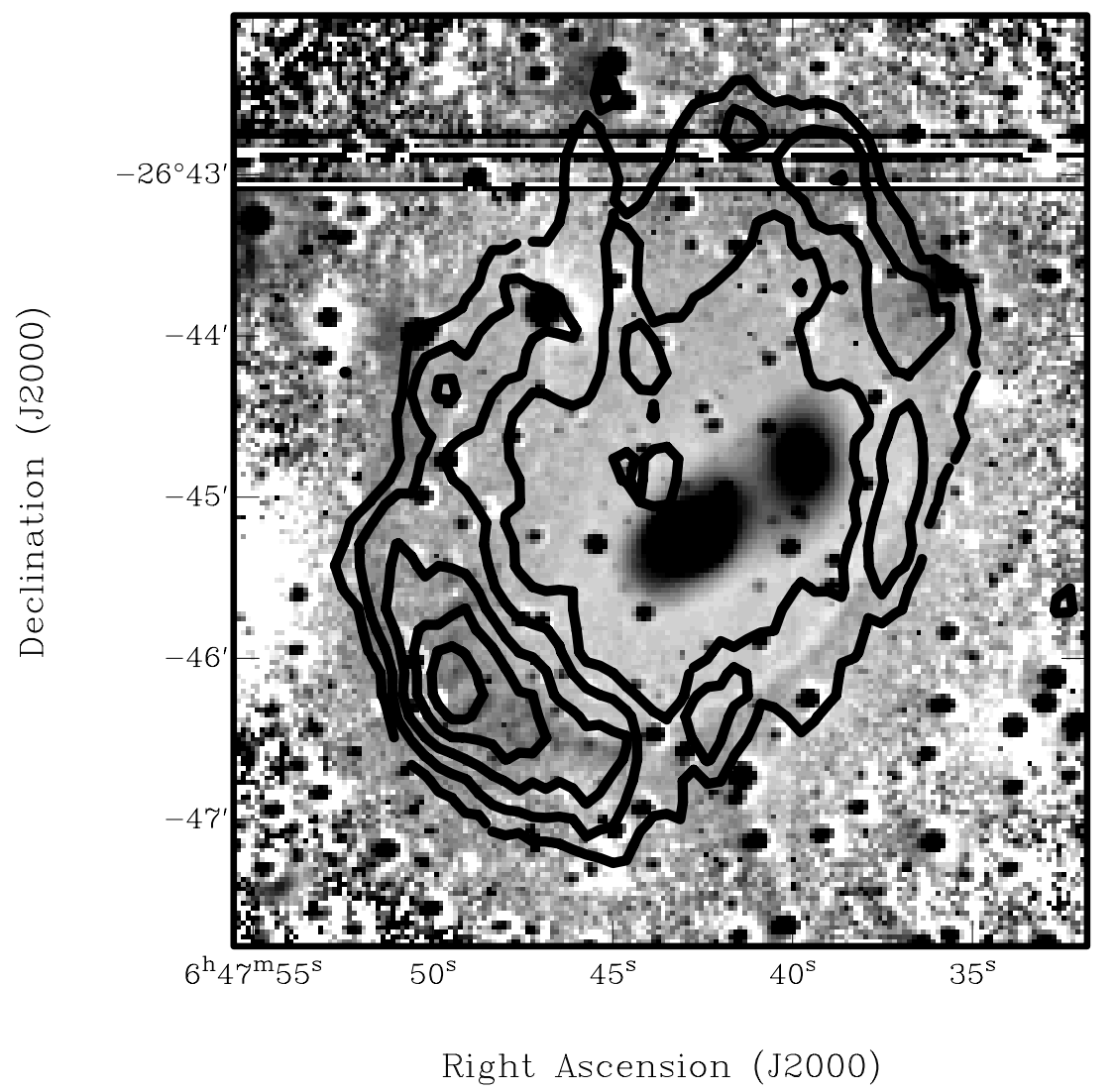

Figure 2- $\mathrm{HI}$ contours (at the same levels as in Figure 1) overlaid on a $B-R$ colour image showing a distinct shelf in the stellar colours associated with the peak Hi column density in the south western arc of the ring.

blue luminosity ratio of only $\sim 6 M_{\odot} / L_{\odot}$, if both galaxies are physically bound and the ring encircles both galaxies. If the ring is actually collapsing onto the galaxies, then the true mass to luminosity ratio is higher; conversely, if the ring is a tidal feature which is no longer bound to the NGC 2292/2293 pair, the mass to luminosity ratio will be lower than that calculated here.

\section{Previously Known Gas Rings}

Hi rings are not common. Briggs (1982) reported the discovery of an elliptical ring of gas enclosing the otherwise normal spiral galaxy NGC 628. Unlike the gas in the LGG 138 ring, the gas in NGC 628 extends from around three times the optical diameter all the way into the optical bulge. The velocity contours of the gas imaged by Briggs (1982) and Shostak \& van der Kruit (1984) suggested the Hi to be distributed in two different orbital planes, with an outer ring of $\mathrm{HI}$ inclined at $\sim 15^{\circ}$ with respect to the inner Hi disk. The total Hi mass of the disk and ring appears to be $(7 \cdot 4 \pm 1.5) \times 10^{9} h_{75}^{-2} M_{\odot}$. Like the LGG 138 ring, NGC 628 is embedded in a group of galaxies, containing three nearby dwarf galaxies. Whilst their luminosities and hence masses indicate that they would have little effect on NGC 628 even in a close encounter (Briggs 1982), it is conceivable that the pair of galaxies UGC 1171 and 1176 were nearby NGC 628 of order 1 Gyr in the past. More recent VLA observations of the system (Kamphuis \& Briggs 1992) have studied the high velocity cloud complexes to the southwest of the galaxy, and an extended $\mathrm{HI}$ arc which may be the tidal remains of an HI-rich object that has merged into the outer disk. Kamphuis \& Briggs (1992) concluded that the galaxy is well modeled by an extended warped disk, and that the HI structure seen in NGC 628 is most likely that of a large isolated system settling towards a flattened disk following many massive accretion events.

An Hi ring that is perhaps more comparable to the LGG 138 ring was found in the M96 region by Schneider (1985) in follow-up observations of an intergalactic cloud found serendipitously by Schneider et al. (1983). The M96 ring has an Hi mass of order $1.5 \times 10^{9} h_{75}^{-2} M_{\odot}$. However, the rotation period of the M96 ring is over ten times that of the LGG $138 \mathrm{ring}$, being $3 \cdot 7 h_{75}^{-1}$ Gyr. Using deep Arecibo observations, Schneider (1985) determined that the ring was composed of several clumps of Hi rather than a single contiguous cloud of HI. The broken ring has projected within it NGC $3379 / 3384 / 3389$ and the faint dwarf galaxy Leo dw $\mathrm{A}$, and extends across $\sim 160 h_{75}^{-1} \mathrm{kpc}$ on the sky at its greatest extent, roughly five times the projected size of the LGG 138 ring. It is the lack of neutral 
hydrogen detected in the galaxies interior to the projected M96 ring which make a comparison with the LGG 138 ring worthwhile. Furthermore, both rings exhibit an $\mathrm{HI}$ mass typical of that bound to an early type spiral galaxy, and very low, if any, luminosity is associated directly with the ring. Even though the M96 ring is disjoint, its regular structure suggests it has probably existed for at least one full revolution, and more likely a few full revolutions ( 10; Schneider 1989).

A third example of an $\mathrm{HI}$ ring is the flat ring surrounding the elliptical galaxy IC 2006. A faint ring of stars around this galaxy prompted Schweizer, van Gorkom \& Seitzer (1989) to obtain VLA images of the Hi emission associated with the galaxy, in the hope that the discovery of an Hi ring or disk would lead to the first confident determination of the mass to light ratio of an elliptical galaxy. The ring has a radius of $13 h_{75}^{-1} \mathrm{kpc}$ at a distance of $18 \cdot 5 h_{75}^{-1} \mathrm{Mpc}$, and an Hi content of at least $3 \times 10^{8} h_{75}^{-2} M_{\odot}$. Under the assumption that the ring is intrinsically flat and circular, kinematical models show the system to have a dark halo containing of order twice as much mass as the luminous matter indicates to be enclosed by the ring. Schweizer, van Gorkom \& Seitzer (1989) suggested that the gas in the ring has been gradually accreted from the companion galaxy (ESO359-G005 at a projected distance of $39 h_{75}^{-1} \mathrm{kpc}$ ), or instead is the result of a Spitzer-Baade, or gas-sweeping, collision. For the latter proposal, the ring would be in a state of expansion at present, and consequently the mass to light ratio determined by Schweizer, de Vaucouleurs \& de Vaucouleurs (1989) would be overestimated. Franx, van Gorkom \& de Zeeuw (1994) presented new Hi data for the ring, and detected Hi smoothly distributed around the entire ring, i.e. it is not broken. Analysis of the (very regular) velocity structure of the ring shows that the galaxy is embedded in a nearly axisymmetric massive halo.

The IC 2006 ring bears a remarkable similarity to the LGG 138 ring: the radii of the two rings, their estimated rotation speeds, and their narrow annular widths are comparable. Furthermore, while the LGG 138 ring contains nearly ten times as much neutral gas as IC 2006, the Hi mass to blue luminosity ratios of the two systems are nearly identical, and both rings have tenuous luminous tails, or even spiral arms, extending into the Hr ring.

\section{Origin of the LGG 138 Ring}

\section{Merging and Shocking in NGC 2292/2293 Pair}

It is tempting to conclude from the optical data alone that the galaxies NGC 2292 and 2293 are interacting, and possibly merging to form a luminous elliptical galaxy over a period of order one Gyr. Indeed, Corwin, de Vaucouleurs \& de Vaucouleurs (1985) listed these two galaxies as an interacting pair, with plumes and loops visible in NGC 2292, and a knotty corona with dust lanes in NGC 2293. Their close proximity on the sky within a common luminous envelope, and their nearly equaland bright - magnitudes are highly suggestive of a collisional merger that is generating the tidal features visible in a high-contrast Digitized Sky Survey (DSS) image of the pair. The ring of neutral hydrogen enclosing both optical bulges lends further weight to the idea that the association is physically real. Finally, the exact coincidence of the neutral gas ring with the break in optical colour of the system in the southwest quadrant of the ring, is strongly suggestive of gas compression and shocking at the radius of the ring, leading to distinctly different stellar populations either side of the shock. The slightly bluer population of stars seen to the outer side of the ring is consistent with star formation occurring at the leading edge of an expanding ring density wave.

Expansion and cooling of a gas envelope or ring, and subsequent compression via shocking, are normal processes during mergers. Spiral galaxy mergers are known to convert a large fraction of the interstellar gas in the participant galaxies into new stars during intensive star formation periods. For example, the Cartwheel ring galaxy shows a strong anti-correlation between $\mathrm{HI}$ and $\mathrm{H} \alpha$ surface densities in the outer ring, leading Higdon (1996) to conclude that the depression of $\mathrm{HI}_{\mathrm{I}}$ in parts of the ring has been caused by the consumption of the gas supply by star formation, which is occurring on the leading edge of a gaseous ring density wave. In light of this observation, it is interesting to note that the southwestern edge of the LGG 138 ring which exhibits the optical colour break coincides with the weakest Hi emission detected from the gas ring. The Cartwheel ring galaxy is generally believed to have its unusual structure as a result of a past collision with a gas-rich galaxy. Milder star-bursts are seen in a number of systems where the interaction is drawn out over a long period of time. For example, the interacting disk-disk system NGC 520 appears to have recently undergone mild star formation throughout both bulges and one of the disks (Stanford \& Balcells 1990; Stanford 1991).

If the galaxies are bound members of the LGG 138 group, as seems likely, then their radial velocity difference can be attributed to peculiar motions within the group. In this case, for NGC 2292 and 2293 to form a bound pair, their physical separation $r$ must be constrained to be not much more than what is seen projected on the sky:

$$
r<\frac{2 G M}{v_{\text {escape }}^{2}}=20 h_{75}^{-1} \mathrm{kpc},
$$

where $M$ is the mass interior to $r$ for, say, NGC 2293 , which has as an upper limit equal to the mass 
interior to the ring $\left(2 \cdot 7 \times 10^{11} h_{75}^{-1} M_{\odot}\right)$, and the escape velocity $v_{\text {escape }}$ is set to $340 \mathrm{~km} \mathrm{~s}^{-1}$. This maximum separation is on the order of the radius of the ring, so if NGC 2292 and 2293 form a bound pair, they are indeed physically nearby each other. It is difficult to imagine how the Hi ring could survive in such a system where the three orbits (of the two galaxies and the ring) are so similar in scale, and probably inclination. That is, over the period of a few orbits, the Hi ring would be distributed into a common halo, or at the very least dramatically disturbed by the galaxies approaching and perhaps crossing the ring. Future numerical modelling would be worthwhile to establish the geometric structure of the system. Combined with the normal appearance of the galaxy nuclei, and the lack of $\mathrm{X}$-ray or radio continuum emission from the nuclei of the galaxies, it is probably unlikely that the two galaxies NGC 2292 and 2293 are currently merging.

Conversely, if the galaxies are not bound members of the LGG 138 group, and some component of their differential velocity is due to smooth Hubble flow, the natural conclusion is that NGC 2292 is physically behind NGC 2293 by $\sim 5 \mathrm{Mpc}$, and it is merely by chance that NGC 2292 is projected within the $\mathrm{HI}_{\mathrm{I}}$ ring. An interesting but difficult measurement to make to shed light on the front-toback ordering of the galaxies would be to look for absorption in the NGC 2293 system of a spectral line emitted at the red-shift of NGC 2292, or vice versa. The presence of such an absorption feature would immediately determine the ordering of the two galaxies. Undoubtedly it would be fascinating if NGC 2292 was found to be in front of NGC 2293, for then they must certainly both be members of the LGG 138 group, and may well collide in the next $\sim 10$ Gyr.

One final remark worth making is that the original survey which lead to the discovery of the LGG 138 ring was partly motivated by the striking images of shells in giant field ellipticals reported by Malin \& Hadley (1997). It is conceivable that if the galaxies NGC 2292 and 2293 are in fact merging, then their product may well be a field elliptical similar to the Malin \& Hadley (1997) examples. Further optical imaging, including 'stacking' of sky survey plates, may be worthwhile to pursue this possibility.

\section{Non-merging Scenario}

A simpler explanation for the Hi ring encircling the NGC 2292/2293 pair is that one of the galaxies was formed with a primordial $\mathrm{HI}$ ring of the type seen in a number of S0/S0a spirals (van Woerden, van Driel \& Schwarz 1983), and the other galaxy is not physically nearby the ring galaxy, but is projected within the ring because of the line of sight. This model will be referred to as the 'non-merging' scenario, but does not preclude any interaction with the more distant NGC 2295 galaxy. The two main arguments in favour of this scenario are that the radial velocities of the two galaxies differ substantially, i.e. by $\sim 340$ $\mathrm{km} \mathrm{s}^{-1}$; and that the ring appears to be connected and circumferentially smooth, with the exception of the bulk concentration to the southeast, coincident with the faint luminous arm or tidal tail. The most likely galaxy to be associated with the Hi ring is NGC 2293, since it appears to be physically closer to the projected centre of the ring, is considerably closer in central radial velocity to the ring, and is mildly more spiral-like than NGC 2292 - see Figure 1. The parameters of the ring - viz. a physical radius of order $15 h_{75}^{-1} \mathrm{kpc}$, a circular speed of $280 \mathrm{~km} \mathrm{~s}^{-1}$ and an orbital period of $\sim 0.3 \mathrm{Gyr}$ - are typical of those for the gas rings in the S0 (lenticular) galaxies observed by van Woerden, van Driel \& Schwarz (1983). Furthermore, most of the rings found by van Woerden, van Driel \& Schwarz (1983) show similar peak column densities $\left(\sim 10^{21} \mathrm{~cm}^{-2}\right)$ to that in the LGG 138 ring, and lack central concentrations of HI.

The two principal models for the formation of a ring around a single galaxy are: an extended and warped primordial gas disk that fails to collapse completely, leaving a system with inner and outer HI disks, possibly having substantially different inclination angles; and the accretion of gas from close interactions or glancing collisions with smaller gas-rich galaxies. For the former, the inner disk is depleted by star formation, while the outer disk barely reaches the critical threshold to form stars - in cases where it does reach critical density over a large part of the ring, the resultant galaxy may become polar ring-like. However, it is difficult to imagine how the colour break seen at the position of the Hr ring could arise without external intervention by an intruder galaxy or potential, and a gas accretion model is more able to explain the uneven structure of typical Hi rings via the capture and tidal stretching of gas-rich dwarf galaxies. Formation of a ring via gas accretion is discussed below. 'Broken' rings may survive for up to a few orbital periods, say $\sim 1$ Gyr, before losing their structure through friction and tidal action to become smooth in appearance.

If the non-merging model is correct for the LGG 138 ring, then the mass to light ratio estimated in Section 2 should be revised accordingly. The rotation speed of the ring implies a total mass within the ring of $2.7 \times 10^{11} h_{75}^{-1} M_{\odot}$, and NGC 2293 has a total blue magnitude of $11 \cdot 7$. Thus the total mass to blue luminosity ratio within the ring is $11 M_{\odot} / L_{\odot}$, provided NGC 2292 is not part of the system, and is instead a background galaxy projected to lie within the ring. This is within the normal range for S0/S0a galaxies (Roberts \& Haynes 1994). 
Gas-sweeping Collision with NGC 2295

It appears that NGC 2292 and 2293 are not merging, and probably not physically related, except that they may be bound members of the LGG 138 group, but not bound to each other. Whilst the ring of $\mathrm{HI}$ may then be a primordial feature associated with the formation of NGC 2293, it is possible that the ring has other origins. The nearby galaxy NGC 2295 has a heliocentric radial velocity of $1823 \pm 60$ $\mathrm{km} \mathrm{s}^{-1}$ (Bottinelli et al. 1992), and is projected at a distance of $\sim 30 \mathrm{kpc}$ from the NGC 2292/2293 pair. This galaxy was not detected in Hi emission, yet is a 13.6 mag Sab spiral well within the spatial and spectral field of view of the ATCA. The absence of gas in NGC 2295 is highly suggestive that the gas has been removed from the galaxy in some way. Corwin et al. (1985) report the detection of a very faint plume to NGC 2292/2293 from NGC 2295. Has NGC 2295 swept past NGC 2293 and been stripped of its gas disk and outer stellar population?

One way to help determine if this is the case would be to compare the sense of rotation of the HI ring with that of the nucleus of the central galaxy. If the angular momentum vectors of the Hi ring and the bulge and disk stellar populations are directed away from each other, then the case for a past gas stripping event is strengthened.* This is because there is no known mechanism by which the gas and stellar components of an isolated spiral that formed normally and has had no interaction with other galaxies can rotate in opposite directions. Recent studies (e.g. Kuijken, Fisher \& Merrifield 1996; Bertola, Buson \& Zeilinger 1992) have shown that at least 10 per cent of S0 galaxies have disks which counter-rotate relative to the stellar components; and it is generally thought that these gas disks result from the capture and tidal stretching of gas-rich dwarf galaxies by massive early type galaxies. NGC 3626 is a classic example of such a system, and Ciri, Bettoni \& Galletta (1995) suggested that any primordial gas remaining in the galaxy, rotating with the stellar component, will cause the counterrotating gas to fall towards the centre of the galaxy over a short period of time. The Hi ring in IC 2006 also appears to rotate in the opposite sense to the stellar population (Schweizer, van Gorkom \& Seitzer 1989).

The projected displacement of NGC 2295 from the centre of the Hi ring implies a most likely true separation of order $\sim 50 h_{75}^{-1} \mathrm{kpc}$. This distance is consistent with what is expected for a gas-sweeping collision. Moore et al. (1996) showed that by the time the response of a galaxy disk to typical close encounters - specifically, the harassment of a large disk by smaller orbiting galaxies - is visible, the perturbing galaxy is usually $\gtrsim 100 \mathrm{kpc}$ distant from the disk galaxy. Furthermore, the simulations of Moore et al. (1996) showed the gas distribution to commonly form ring structures and tidal tails in the resultant system. The radial velocity of NGC 2295 relative to the ring is $220 \mathrm{~km} \mathrm{~s}^{-1}$, very similar to the maximum observed circular velocity of gas in the ring. Assuming a bound spherical system, this velocity corresponds to a physical relative velocity of $\sim 380 \mathrm{~km} \mathrm{~s}^{-1}$, leading to the conclusion that if NGC 2295 has recently experienced a close encounter with NGC 2293, the interaction was on the order of $100 \mathrm{Myr}$ ago.

\section{Conclusions}

Hr observations of the LGG 138 galaxy group have revealed a $\sim 30 \mathrm{kpc}$ diameter ring of gas projected around two bright spiral galaxies in the group. The ring exhibits regular rotation, and future numerical models may show it to be additionally undergoing expansion or contraction. Deep optical images show a break in stellar colour associated with the southern and south-western parts of the ring. The ring is most likely to have formed either from the tidal accretion of gas from the nearby galaxy NGC 2295, or instead during the perturbed collapse of one of NGC 2292/2293, probably the latter. Future studies will be directed at re-measuring the radial velocities of the galaxies NGC 2292/2293/2295, possibly mapping the rotation of the galaxy nuclei, and searching for CO emission in NGC 2295 to ascertain whether it is likely to have lost Hi gas to the NGC 2292/2293 pair.

\section{Acknowledgments}

I am grateful to L. Staveley-Smith and R. Webster for their scientific contributions, and to M. Brown for acquiring the optical data. I acknowledge the financial support of an Australian Postgraduate Award. The Australia Telescope is funded by the Commonwealth of Australia for operation as a National Facility managed by CSIRO.

\section{References}

Barnes, D. G. 1998, PhD Thesis, Univ. of Melbourne

Bertola, F., Buson, L. M., \& Zeilinger, W. W. 1992, ApJ, 401, L79

Bottinelli, L., Durand, N., Fouque, P., Garnier, R., Gouguenheim, L., Paturel, G., \& Teerikorpi, P. 1992, A\&AS, 93, 173

Briggs, F. H. 1982, ApJ, 259, 544

Ciri, R., Bettoni, D., \& Galletta, G. 1995, Nature, 375, 661

Corwin, H. G., de Vaucouleurs, A., \& de Vaucouleurs, G. 1985, Southern Galaxy Catalogue, Univ. Texas Monographs in Astronomy

Fouqué, P., Gourgoulhon, E., Chamaraux, P., \& Paturel, G. 1992, A\&AS, 93, 211

\footnotetext{
* The converse is not true of course - aligned or nearly aligned angular momentum vectors do not rule out gas stripping of a nearby companion.
} 
Franx, M., van Gorkom, J. H., \& de Zeeuw, T. 1994, ApJ, 436,642

Garcia, A. M. 1993, A\&AS, 100, 47

Garcia, A. M. 1995, A\&A, 297, 56

Hickson, P. 1982, ApJ, 255, 382

Higdon, J. L. 1996, ApJ, 467, 241

Kamphuis, J., \& Briggs, F. 1992, A\&A, 253, 335

Koribalski, B. 1996 in Minnesota Lectures on Extragalactic Hi, ed. E. Skillman (San Francisco: PASP), p. 238

Kuijken, K., Fisher, D., \& Merrifield, M. R. 1996, MNRAS, 283,543

Lauberts, A., \& Valentijn, E. 1989, The Surface Photometry Catalogue of the ESO-Uppsala Galaxies (Garching: European Southern Observatory)

Malin, D., \& Hadley, B. 1997, PASA, 14, 52

Moore, B., Katz, N., Lake, G., Dressler, A., \& Oemler, A., Jr 1996, Nature, 379, 613

Nolthenius, R. 1993, ApJS, 85, 1
Roberts, M. S., \& Haynes, M. P. 1994, ARA\&A, 32, 115

Rose, J. A. 1977, ApJ, 211, 311

Schneider, S. E. 1985, ApJ, 288, L33

Schneider, S. E. 1989, ApJ, 343, 94

Schneider, S. E., Helou, G., Salpeter, E. E., \& Terzian, Y. 1983, ApJ, 273, L1

Schweizer, F., van Gorkom, J. H., \& Seitzer, P. 1989, ApJ, 338,770

Shostak, G. S., Sullivan, W. T., \& Allen, R. J. 1984, A\&A, 139,15

Shostak, G. S., \& van der Kruit, P. C. 1984, A\&A, 132, 20

Stanford, S. A. 1991, ApJ, 381, 409

Stanford, S. A., \& Balcells, M. 1990, ApJ, 355, 59

van Woerden, H., van Driel, W., \& Schwarz, U. J. 1983, in Internal Kinematics and Dynamics of Galaxies, ed. E. Athanassoula, IAU Symp., 100, 99

Williams, B. A., \& van Gorkom, J. H. 1988, AJ, 95, 352 